\title{
PRIMARY SPINAL CORD GLIOBLASTOMA IN YOUNG POPULATION; REPORT OF TWO CASES
}

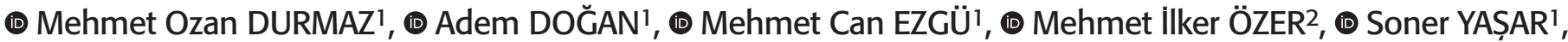 (1) Alparslan KIRIK ${ }^{1}$}

\author{
${ }^{1}$ Gülhane Training and Research Hospital, Clinic of Neurosurgery, Ankara, Turkey \\ ${ }^{2}$ Republic of Turkey Ministry of Health, Dr. Yasar Eryılmaz State Hospital, Clinic of Neurosurgery, Ağrı, Turkey
}

Primary spinal glioblastoma is a very rare disease of spinal cord with high morbidity and mortality. In this study, we presented two patients surgically treated for primary spinal glioblastoma.

The first case was a 21-year-old male who has a lesion at T9-T10 spinal level in Magnetic resonance imaging (MRI) in which subtotal resection was performed.

The second case was a 22-year-old male patient who has a lesion in the spinal canal between the level of C3 andT1 in MRI. The lesion was resected subtotally.

The pathological diagnosis was reported as glioblastoma in both cases. Primary spinal glioblastoma is a very rare disease. Because of its clinically and radiologically malignant behavior of prognosis, this disease must be kept in mind in differential diagnosis of cervical-thoracal spinal cord pathologies and early diagnosis and treatment should be started as soon as possible.

Keywords: Primer spinal cord tumor, glioblastoma, young patient

\section{INTRODUCTION}

Glioblastoma is the most common and most aggressive primary malignant brain tumor in adults. On the other hand, glioblastoma with primary spinal involvement is very rare ${ }^{(1)}$. Spinal glioblastomas have an incidence of $1-5 \%$ in all glioblastoma cases and $1.5 \%$ of all spinal tumors ${ }^{(2)}$. More than $60 \%$ of cases are in the cervical or cervicothoracic region(3). The purpose of this case report is to provide basic information about this rare disease to present and discuss in the context of the literature.

\section{CASE REPORTS}

\section{Case 1}

First case was a 21-year-old male who was referred to our clinic with a complaint of weakness and sensory loss in the lower extremity that started 1 month ago. Urinary incontinence occurred three days after his first admission to hospital. Neurological examination revealed paraplegia and hypoesthesia below T10 level. Deep tendon reflexes were hypoactive.
As a result of the cranial and spinal Magnetic resonance imaging studies, intramedullary mass was found at T9- T10 level (Figure 1A). Bilateral tibial Sensorimotor Evoked Potential (SEP) cortical response could not be obtained. The patient was operated and the mass was subtotally resected. Postoperative neurological findings were same after surgery.

Postoperative histopathological examination showed an infiltrative tumor of the medulla spinalis. The tumor was found to be formed by atypical glial cells with large hyperchromatic nuclei. Highly mitosis and geographic necrosis areas were detected in pathologic specimen. Neoplastic cells were marked with glial fibrillary acidic protein (GFAP). Glioblastoma was diagnosed with these morphologic and immunohistochemical findings (Figure 1B).

Following postoperative wound healing, chemotherapy and radiotherapy were applied to the patient. After chemoradiotherapy, the patient was admitted to a rehabilitation program for motor weakness. Control MRI was performed 3 months after surgery. Local recurrence or distant metastasis was not detected following chemoradiotherapy. Neurological findings were not different from those in early postoperative period in follow up. 
turkishspine

Local recurrence and distant metastasis were not detected in the spinal MRI at the sixth month of surgery. At the ninth month of surgery, new lesions were observed on the anterior horn of right lateral ventricle and T7-8 vertebrae in the control $\mathrm{MRI}$, in addition any surgical treatment was required for these lesions (Figure 1C,D). Surgical treatment was not considered for cranial lesion because the patient's neurological status did not show any deterioration in neurological examination after ninth months of surgery. For the spinal recurrence, it was thought that a second operation would not provide an additional benefit to the patient's outcome. The patient was directed to radiation oncology clinic for radiotherapy. He received radiotherapy for

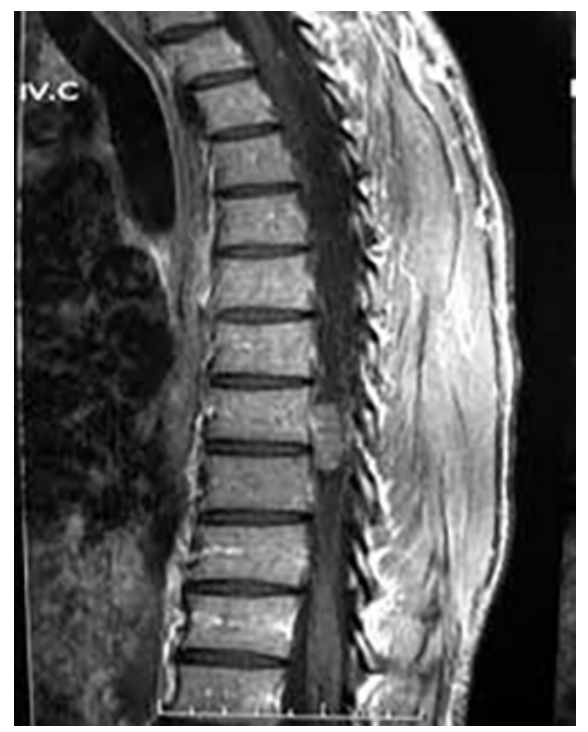

Figure 1A. Preoperative thoracolumbar T1 sagittal contrast MR image

MR: Magnetic resonance

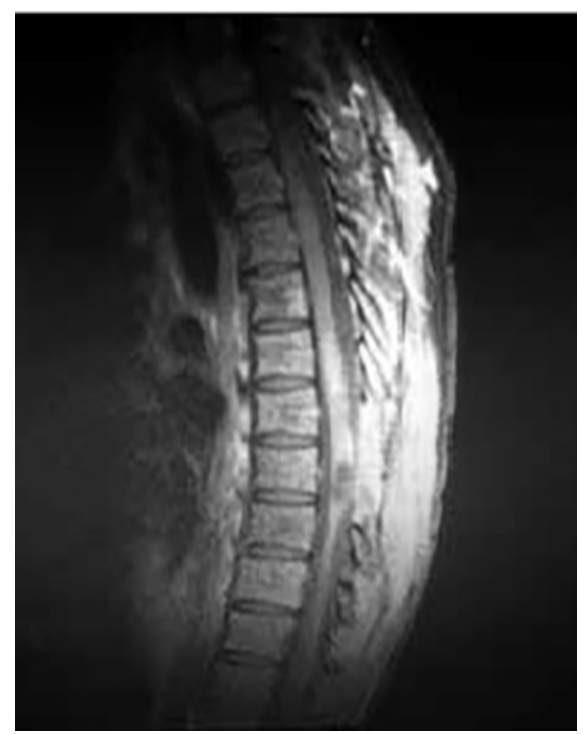

Figure 1C. Postoperative $9^{\text {th }}$ month thoracolumbar T2 sagittal contrast MR image

MR: Magnetic resonance the cranial lesion and was discharged afterwards. It is known that the patient died about 16 months after being diagnosed.

\section{Case 2}

Second case was a 22-year-old male patient, referred to our clinic with the complaints of right sided numbness and weakness in extremities for 2 months. There was not any obvious pathology found on systemic examination. Right sided $1 / 5$ hemiparesis was observed. Babinski's reflex was positive on the right side. Deep tendon reflexes were hyperactive in the lower limbs.

An intramedullary expansile mass was revealed on the spinal cord with an extending of C3 to T1 vertebrae spinal levels. In $\mathrm{MRI}$, the size of the tumor was measured as $14 \times 18 \times 65 \mathrm{~mm}$ (Figure 2A). The lesion was thought to be compatible with

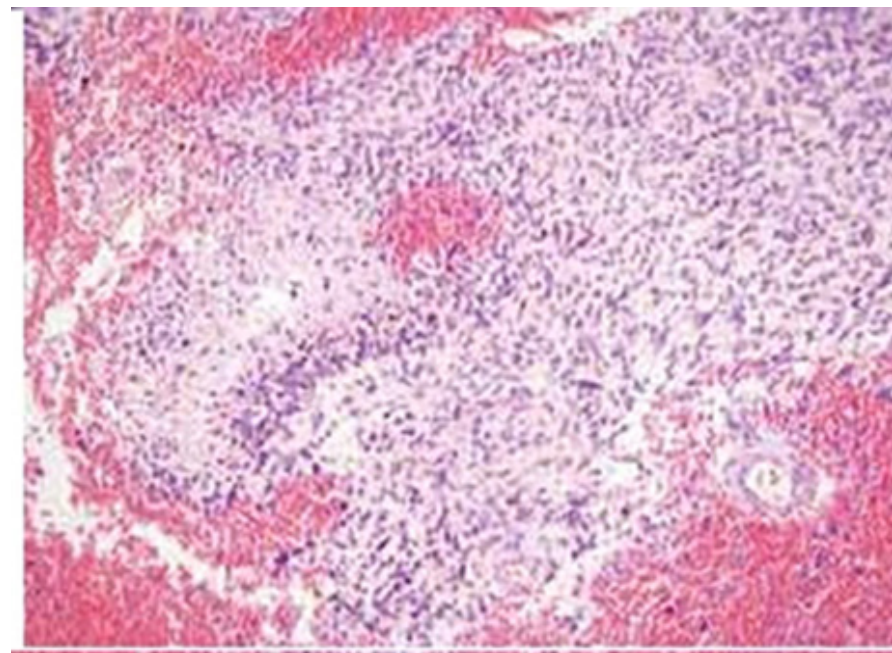

Figure 1B. Palizadic alignment around the necrotic area histological appearance of the tumor (x200, HE), HE: Hematoxylin and eosin

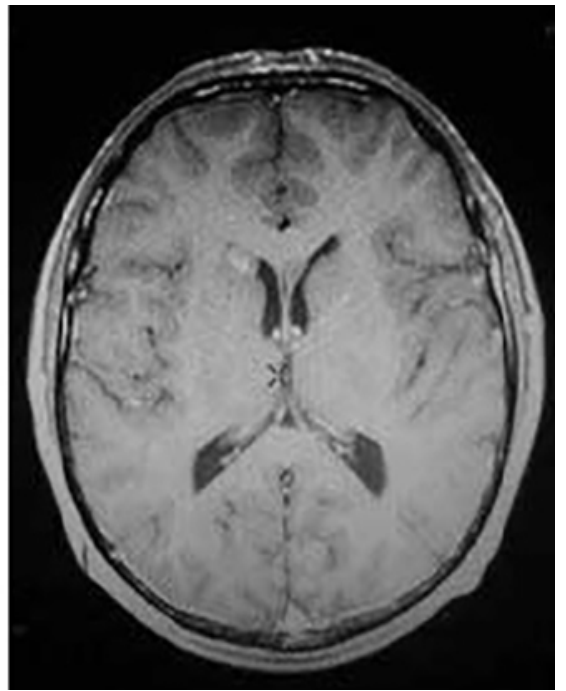

Figure 1D. Postoperative $9^{\text {th }}$ month cranial T1 axial contrast MR image

MR: Magnetic resonance 
ependymomas or astrocytomas due to mild degree of contrast enhancement. An edematous appearance was observed at the inferior contour of the lesion. The patient had additional thoracolumbar and brain MRI scans and no other involvements were detected.

The extension of right tibial nerve latency was detected in SEP. Soft-grained mass was subtotally resected with C4-6 total laminectomy. In the post-operative early neurologic examination, lower extremities and right upper extremity were plegic, left upper extremity was $4 / 5$ paretic, patient was anesthetic under C4 level, and lower extremity reflexes were hyperactive. The patient was operated in the presence of neuromonitoring. Bilateral lower extremity response was not obtained in motor evoked potential during surgery.

Histopathological examination revealed that the tumor was composed of atypical glial cells with hyperchromatic nucleus, pleomorphic and high mitotic activity on a fibrillary

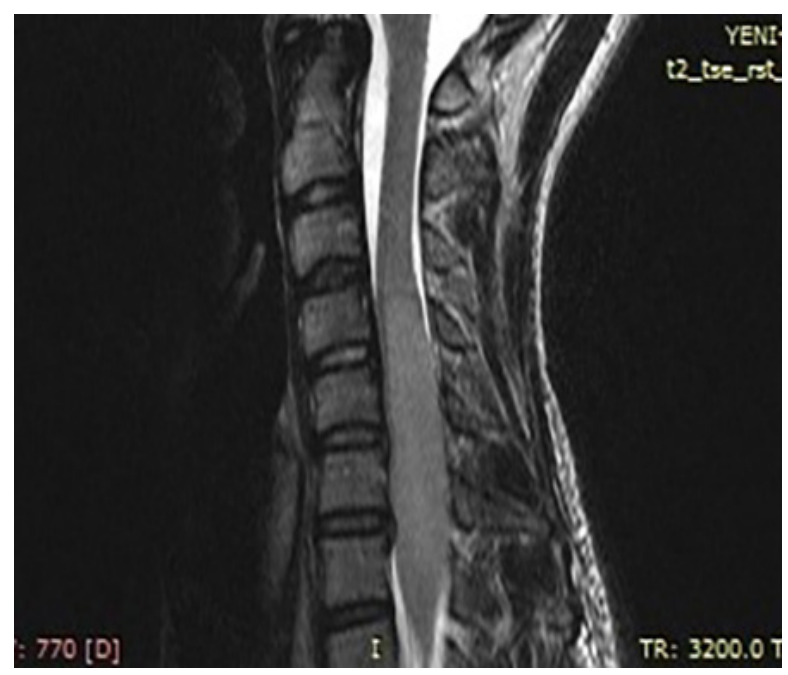

Figure 2A. Preoperative thoracolumbar T2 sagittal contrast MR image

MR: Magnetic resonance

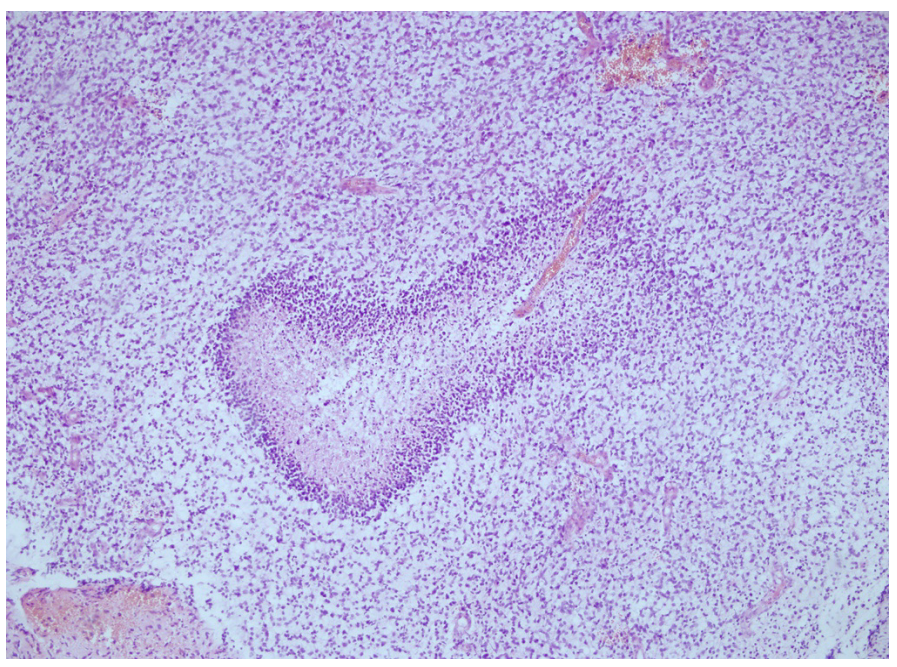

Figure 2B. Tumor necrosis area (H\&E, x40)

H\&E: Hematoxylin and eosin stain site. Necrosis foci and microvascular proliferation areas were present around the tumor, which was palindically dyed (Figure 2B). Immunohistochemical examination revealed that the tumor was diffusely and strongly labeled with GFAP, and the cells showed nuclear positivity with P53. Ki-67 proliferation index was $70-75 \%$ (Figure 2C). Significant immunoreactivity was not detected with neuron-specific enolase, synaptophysin, chromogranin and monofilament. Tumor was reported as gliblastoma (WHO grade IV)

In the postoperative first week, the right median nerve response was not obtained in control SEP, whereas the right tibial nerve response latency was long. Left median and tibial nerve responses were normal. He underwent physiotherapy 1 month after surgery and tetraparesis recovered partially (Figure 2D). The patient was directed to the radiation oncology clinic for further treatment. After radiotherapy, adjuvant chemotherapy (temozolomide) was given to the patient. The patient was

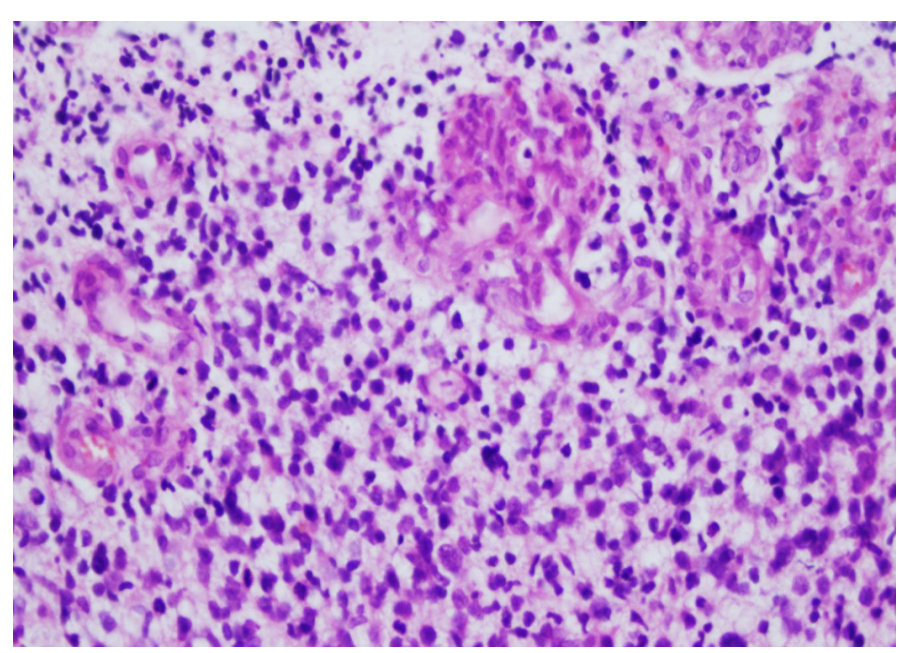

Figure 2C. Microvascular proliferation area in tumor (H\&E, x100) H\&E: Hematoxylin and eosin stain

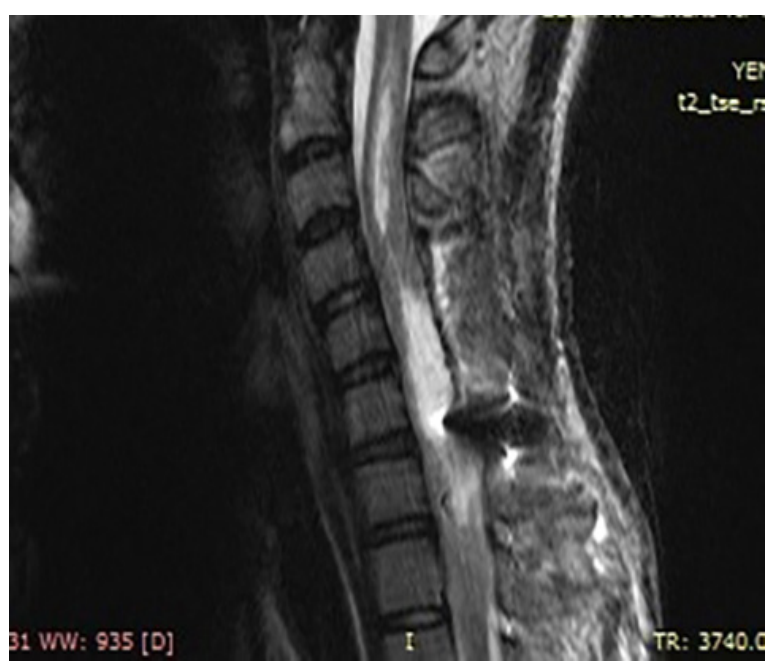

Figure 2D. Postoperative first month thoracolumbar T2 sagittal contrast MR image

MR: Magnetic resonance 
transferred to a physical therapy center after radiotherapy. After about two months of physical therapy, the patient was discharged. It was learned that the patient died about 5.5 months after diagnosis.

\section{DISCUSSION}

Glioblastoma (GBM) is the most common primary malignant brain tumor in adults. In spite of that, spinal intramedullary astrocytomas constitute approximately $1 \%$ of all central nervous system tumors and $6-8 \%$ of all spinal cord tumors. Glioblastoma accounts for $7.5 \%$ of all intramedullary gliomas and only $1.5 \%$ of all spinal tumors ${ }^{(4)}$. It has been reported in the literature that spinal glioblastomas usually occur in the second and third decades, frequently in the cervical region followed by the cervicothoracic region ${ }^{(5,6)}$. Similarly, our cases were in the second decade and the settlement was at the cervical and thoracic spinal levels. Shen et al.(6) showed a review of the literature, and to date, a total number of 165 primary spinal GBM cases have been reported since 1938.

Although spinal glioblastomas are seen as primaries, spinal extension of cerebral primer tumors may also occur. Glioblastoma is rarely seen in the differential diagnosis of spinal cord masses, but should be considered in diagnosis. After diagnosis, all spinal column and brain should be scanned with MRI. Primary development or metastasis of the tumor should be discriminated and the treatment algorithm should be determined accordingly ${ }^{(6)}$.

It may be difficult to diagnose early because the initial symptoms of the disease are not apparent. Neurological deterioration occurs rapidly. Diagnosis can be made by the presence of various deficits such as motor and sensory loss, hyperactive reflexes, sexual dysfunction, bladder and bowel function disorders in the clinic progress of disease ${ }^{(4)}$. The most common finding is motor weakness seen in $87 \%$ of spinal glioma cases. The 5 -year survival rates are in these patients with a poor rate of $0 \%$ to $33 \%$. Right after the diagnosis, the survival rates range from 6 to 16 months ${ }^{(7)}$.

Today, MRI is the ideal imaging method for diagnosis ${ }^{(8,9)}$. In MRI, the mass is usually seen as iso-hypointense with spinal cord on T1-weighted images and hyperintense on T2-weighted images. Cystic areas within the tumoral tissue are frequently seen. Irregular homogeneous contrast retention and expansions of the spinal cord by neoplasm are other common findings.

Leptomeningeal involvement is seen in $23-27 \%$ of autopsy series of cerebral glioblastoma cases ${ }^{(10,11)}$. These metastases are generally thought to originate from neoplastic cells carried by the cerebrospinal fluid ${ }^{(2,12)}$.

The aim of the surgery of high grade glial tumors is primarily maximal resection of the tumor. Recurrence of the disease is common even if total resection is performed by surgery. In high grade glial tumors, recurrences frequently develop around the origin of the lesion. In $58 \%$ of high grade glioma cases, tumor spread is seen throughout the subarachnoidal space ${ }^{(13)}$.
Spinal glioblastoma treatment includes surgical resection and adjuvant radiotherapy. The application of radiotherapy and chemotherapy is important because it contributes to the enhancement of disease control ${ }^{(14)}$.

To increase free survival rates in these tumors, simultaneous use of temozolamide $\left(75 \mathrm{mg} / \mathrm{m}^{2}\right.$, adjuvant temozolamide $200 \mathrm{mg} /$ $\mathrm{m}^{2}$ daily for 6 months, followed by 28 days) was emphasized in the study of Stupp et al. in 2005(15,16).

Both of our cases underwent simultaneous radiotherapy after surgery and adjuvant chemotherapy was applied afterwards. Spinal cord primary glioblastoma is still a malignant neoplasm with high mortality and morbidity despite surgical treatment and other adjuvant therapies. It is very important to initiate appropriate treatment by making correct diagnosis in the shortest possible time in glioblastoma, if the mean survival rate of 15 months is considered in this pathology $y^{(4)}$. Today, glioblastoma, which has started to differ in terms of settlement location and frequency, is at the top of the list of increasingly frequent differential diagnoses among primary spinal cord pathologies.

\section{Ethics}

Informed Consent: Retrospective study.

\section{Authorship Contributions}

Surgical and Medical Practices: M.O.D., A.D., M.C.E., M.I.Ö., S.Y., A.K., Concept: M.O.D., M.C.E., Design: M.O.D., M.C.E., S.Y., Data Collection or Processing: M.O.D., M.C.E., S.Y., Analysis or Interpretation: A.D., M.O.D., M.I.Ö., M.C.E., A.K., Literature Search: A.D., M.O.D., M.C.E., A.K., Writing: A.D., M.O.D.

Conflict of Interest: No conflict of interest was declared by the authors.

Financial Disclosure: The authors declared that this study received no financial support.

\section{REFERENCES}

1. Scarrow AM, Rajedran P, Welch WC, Glioblastoma multiform of the conus medullaris. Clin Neural Neurosurg. 2000; 102:166-7.

2. Andrews AA, Enriques L, Renaudin J, Tomiyasu U. Spinal intramedullary glioblastoma with intracranial seeding. Report of a case. Arch Neurol. 1978;35:244-5.

3. Cooper PR. Outcome after operative treatment of intramedullary spinal cord tumors in adults: intermediate and long-term results in 51 patients. Neurosurgery. 1989;25:855-9.

4. Ciapetta P, Salvati M, Capoccia G, Artico M, Raco A, Fortuna A. Spinal glioblastomas: report of seven cases and review of the literature. Neurosurgery. 1991;28:302-6.

5. Caroli E, Salvati M, Ferranate L. Spinal glioblastoma with brain relapse in child: Clinical considerations. Spinal Cord. 2005;43:565-68.

6. Shen CX, Wu JF, Zhao W, Cai ZW, Cai RZ, Chen CM. Primary spinal glioblastoma multiforme, A case report and review of the literature. Medicine (Baltimore). 2017;96:e6634.

7. Takara $E$, Ide $M$, Yamamato $M$, Imanaga $H$, Jimbo $M$, Imai $M$. A case of the intracranial and spinal dissemination of primary spinal glioma. No Shinkei Geka. 1985;13:301-5.

8. Acıkgöz B, Kalaycı M, Sanser G. Intramedullary spinal cord tumors. Turkey Clinics, J Surg Med Sci. 2007;3:150-6. 
9. Koeller KK, Rosenblum RS, Morrison AL. Neoplasms of the spinal cord and filum terminale: radiologic-pathologic correlation. Radiographics. 2000;20:1721-49.

10. Onda K, Tanaka R, Takahashi H, Takeda N, Ikuta F. Cerebral glioblastoma with cerebrospinal fluid dissemination: a clinicopathological study of 14 cases examined by complete autopsy. Neurosurgery. 1989;25:533-40.

11. YungWA, Horten BC, Shapiro WR. Meningeal glimatosis: a review of 12 cases. Ann Neurol. 1980;8:605-8.

12. O'Connell JE. The subaarachnoid dissemination of spinal tumors. J Neurol Neurosurg Psychiatry. 1946;9:55-62.
13. Gunderson LL, Tepper JE. Spinal Cord Tumors. Chapter 30. In: Siker M.L, Bovi J., Alexander B (Eds.). Clinical Radiation Oncology, Philadelphia 2015; pp:532-3.

14. Nakamura M, Ishii K, Watanabe K, et al. Surgical treatment of intramedullary spinal cord tumors: prognosis and complications. Spinal Cord. 2008;46:282-6.

15. Kim WH, Yoon SH, Kim CY, et al. Temozolamide for malignant primary spinal cord glioma: An experience of six cases and a literature review.J Neurooncol. 2011;101:247-54.

16. Bernadine RD. Tumors of the Central Nervous System, Adult gliomas. jiade J. LUi Luther W. BRADY. Radiation oncology: An Evidence-Based approach 2008;pp:487. 